\title{
Fatal Immune Response Associated with Anti-PD1: Pathological Insight
}

Supari $\mathrm{D}^{1 *}$, Meyer $\mathrm{J}^{2}$, Heindl $\mathrm{U}^{2}$, Karthaus $\mathrm{M}^{3}$, Pohlmann $\mathrm{H}^{3}$, Hann von Weyhern $\mathrm{C}^{1}$ and Kremer $\mathbf{M}^{1}$

${ }^{1}$ Institute of Pathology, Klinikum Neuperlach, Munich, Germany

${ }^{2}$ Department of Pulmonology, Gastroenterology und Emergency Medicine, Klinikum Harlaching, Munich, Germany

${ }^{3}$ Department of Hematology, Oncology und Palliative Medicine, Klinikum Neuperlach, Munich, Germany

*Corresponding author: Divya Supari, Municipal Hospital of Munich, Klinikum Neuperlach, Oskar-MariaGraf-Ring 52, 81737 Munich, Germany

Received: April 07, 2021; Accepted: May 13, 2021; Published: May 20, 2021

\begin{abstract}
The recent introduction of immune checkpoint inhibitor therapy with antiPD-1 agents has shown encouraging responses in gastrointestinal oncology. However, it is also known to possibly result in uncontrolled T cell autoreactivity, leading to a broad range of immune related adverse events.
\end{abstract}

We present a patient who developed sequential immune-related hematologic and hepatic complications following pembrolizumab therapy for metastatic colon cancer. In particular, we describe in detail the histopathologic and immunohistochemical findings in the liver and bone marrow, adding to the spectrum of morphological changes which may be observed in association with PD-1 inhibitor therapy.

The report also highlights the occurrence of these adverse reactions even after discontinuation of immunotherapy, eventually resulting in the death of the patient.

Keywords: Colon cancer; Immune checkpoint inhibitor therapy; Anti-PD-1; Neutropenia; Hepatitis; Thrombocytopenia; Immune-related adverse events

\section{Case Presentation}

An 85-year-old man was diagnosed in October 2017 with adenocarcinoma of the colon ascendens. He underwent right side hemicolectomy. Pathological examination of the specimen revealed a colon adenocarcinoma NOS with UICC stage IIIA (pT3, pN1a (1/19), pM0, L1, V0), MSI-H. Fourteen months later, in December 2018, surveillance computed tomography (CT) findings showed multiple enlarged retroperitoneal and para-aortic lymph nodes. Subsequently performed lymph node biopsies confirmed metastatic infiltration of colon adenocarcinoma. The patient was subsequently treated with nine cycles of pembrolizumab (200mg every three 3 weeks) from December 2018 to June 2019.

Two weeks after completion of the ninth cycle of pembrolizumab, the patient was hospitalized due to severe febrile neutropenia with an absolute neutrophil count (ANC) of $0 / \mu l$. The serological markers for hepatitis A, B, C, HSV and CMV were tested negative. Blood and urine cultures performed were negative. Bone Marrow (BM) aspiration and trephine biopsy findings are described in detail below. Pembrolizumab was discontinued. The patient was subsequently treated with meropenem (1g daily), Granulocyte Colony-Stimulating Factor (G-CSF) (34 million IU per day) and high-dose oral prednisone (100mg daily). After 4 weeks he had recovery of his neutrophil count with ANC of $2500 / \mu \mathrm{L}$. Other blood counts were within the normal limits.

In early August the patient was diagnosed with invasive pulmonary aspergillosis based on serum Aspergillus galactomannan antigen by enzyme immunoassay (index 1.10) combined with bilateral pulmonary infiltrate on CT images. Intravenous voriconazole followed by oral therapy was initiated. Prior to voriconazole therapy the liver transaminases were mildly elevated with an aspartate aminotransferase (AST) level of 36U/L (normal range: <35U/L) and an alanine aminotransferase (ALT) level of $117 \mathrm{U} / \mathrm{L}$ normal range: $<45 \mathrm{U} / \mathrm{L}$ ). After 10 days of treatment with voriconazole, the transaminases peaked and then gradually decreased with dose reduction of voriconazole (Figure 1). Seven weeks later voriconazole was terminated as his symptoms improved and his CT chest showed a marked resolution of pre-existing aspergillosis associated lesions.

The patient was readmitted in early October for abrupt elevation of liver enzymes with serum ALT 335U/L, AST 452U/L, and total bilirubin 6.3g/dl. The Antinuclear Antibody (ANA) was borderline positive with a few nuclear dots with a titer of 1:160. Antimitochondrial antibody, anti-smooth muscle antibody, anti-liverkidney-microsomal antibody were negative. The serological markers for hepatitis A, B, C, HSV, EBV and CMV were negative. Persistent elevation of liver enzymes raised a suspicion of pembrolizumabassociated hepatitis (Figure 2), a liver biopsy was subsequently performed. Additionally, an isolated thrombocytopenia was noticed with a platelet count of 79/nl (normal range 140-360/nl), for which BM biopsy was repeated. Liver biopsy and BM biopsy findings are described in detail below. The Patient received high dose corticosteroid and mycophenolate for suspected pembrolizumab induced severe hepatotoxicity. Despite this intense immunosuppression, the patient's Liver Function Test (LFT) showed no improvement. Patient gradually deteriorated and died three weeks later due to fulminant hepatic failure.

\section{Histopathological findings}

BM findings of July 2019 (first biopsy 6 months after initiation of pembrolizumab): $\mathrm{BM}$ aspirate and the trephine biopsy from posterior superior iliac spine revealed normocellular marrow with severe reduction of mature neutrophils. It revealed a maturation arrest at the promyelocyte stage. Erythroid cells 


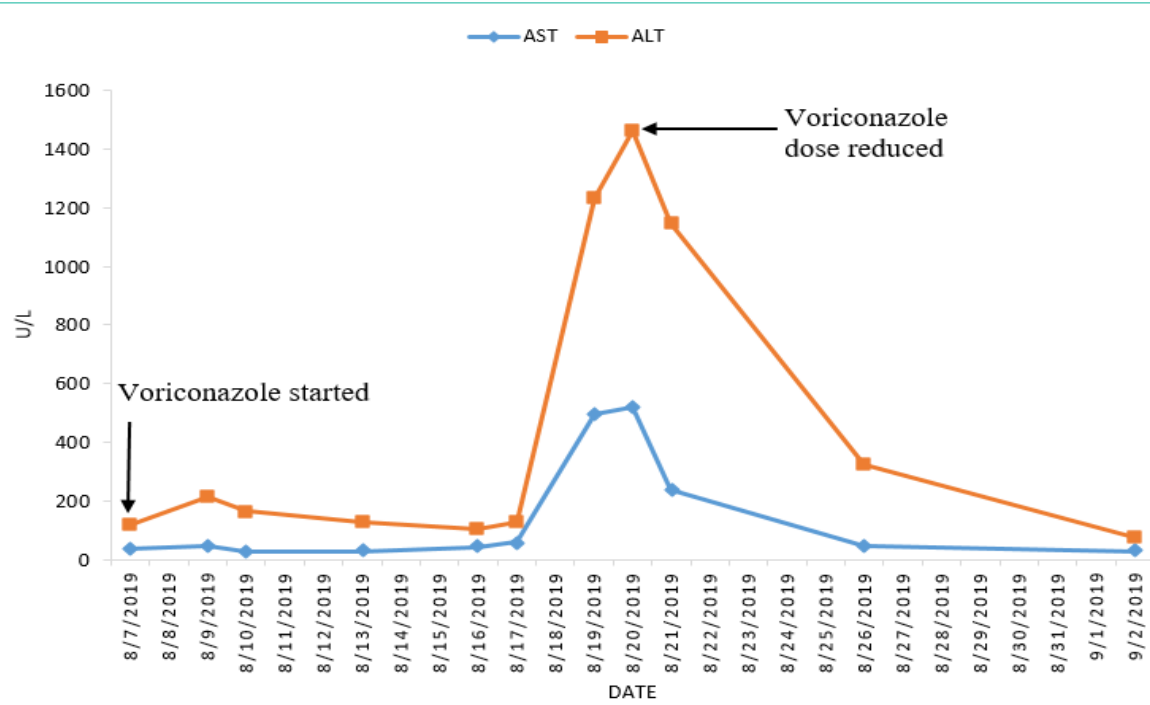

Figure 1: Graph showing trend of serum transaminase levels during voriconazole therapy. Voriconazole therapy was started with a loading dose of $6 \mathrm{mg} / \mathrm{kg}$ every $12 \mathrm{~h}$ i.v on the first day, followed by a maintenance doses of $200 \mathrm{mg}$ p.o twice a day. On August 20,2019 voriconazole dose was reduced to $100 \mathrm{mg}$ p.o twice a day. AST: Aminotransaminase; ALT: Alanine Aminotransaminase.

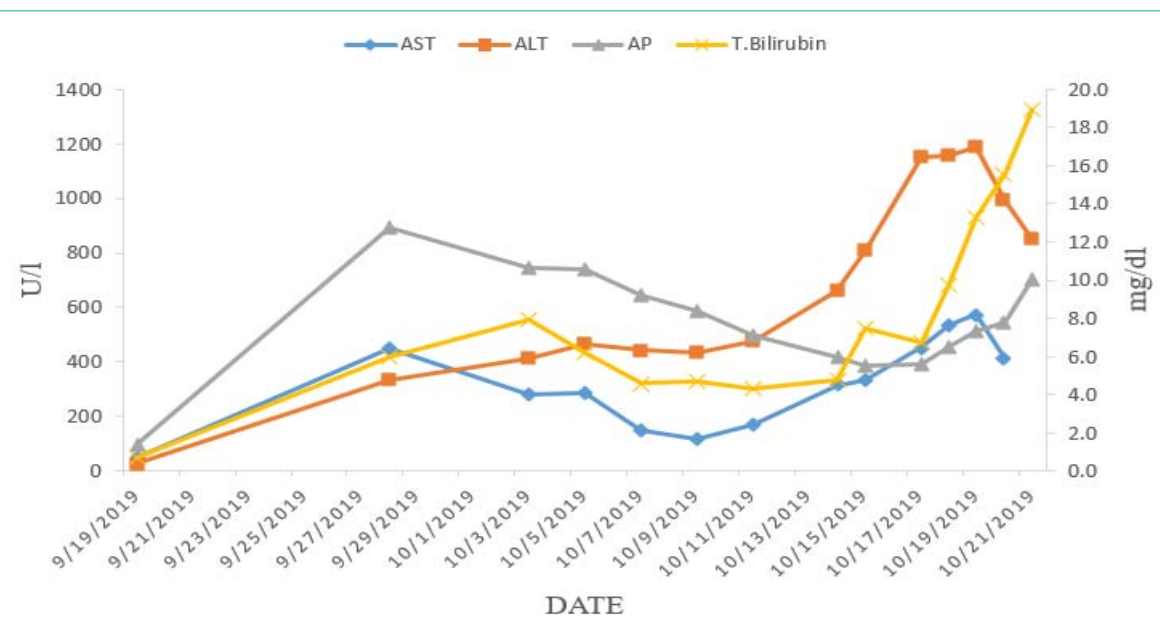

Figure 2: Graph showing trend of Liver function tests prior to and during admission for immune-related hepatitis.

AST: Aspartate Aminotransaminase; ALT: Alanine Aminotransaminase; AP: Alkaline Phosphatase; T. Bilirubin: Total Bilirubin.

showed mild dyserythropoiesis and megakaryocytes were normal in number, morphology and distribution. Flow Cytometric (FC) immunophenotypic studies revealed an increased population of polyclonal T-lymphocytes. Immunohistochemical analysis on trephine showed an admixture of $\mathrm{CD} 3, \mathrm{CD} 4$ and $\mathrm{CD} 8$ positive interstitial $\mathrm{T}$ cells, which were considered as reactive (Figure 3 ).

Liver biopsy of October 2019 (9 months after initiation of pembrolizumab): Liver biopsy of the patient showed a pattern of acute lobular hepatitis. Numerous foci of lymphohistiocytic lobular inflammation with scattered neutrophils, patchy necrosis and acidophilic bodies was observed. There were many noncaseating small and large granulomas. Portal areas showed a mixed inflammatory infiltrate. Occasional plasma cells were seen. Additionally bile ductular proliferation, focal bile duct injury, mild venular endothelitis and mild hepatocellular cholestasis were noted. Immunohistochemistry demonstrated the predominant lobular and portal infiltration of $\mathrm{CD}^{+}$and $\mathrm{CD} 8^{+}$lymphocytes, while fewer $\mathrm{CD} 4^{+}$ and occasional CD20+ lymphocytes were detected (Figure 4).

Second BM biopsy of October 2019 (9 months after initiation of pembrolizumab): Trephine biopsy in the setting of thrombocytopenia showed a hypercellular marrow (70\% Cellular) with trilineage hyperplasia. There were a few foci of non- caseating granulomas with reactive lymphocytosis. Immunohistochemistry revealed that the lymphocytes were predominantly $\mathrm{CD}^{+}$and $\mathrm{CD}^{+}$ $\mathrm{T}$ cells along with a very few $\mathrm{CD} 4^{+} \mathrm{T}$ cells and $\mathrm{CD} 20 \mathrm{~B}^{+}$cells (Figure 5). FC done on a BM aspirate also showed a similar phenotype an increased population of $\mathrm{CD}^{+}$and $\mathrm{CD} 8^{+}$lymphocytes.

\section{Discussion}

Immune-Related Adverse Events (irAEs) caused by pembrolizumab monotherapy is known to occur less frequently and are often less severe compared are combination therapy with either 

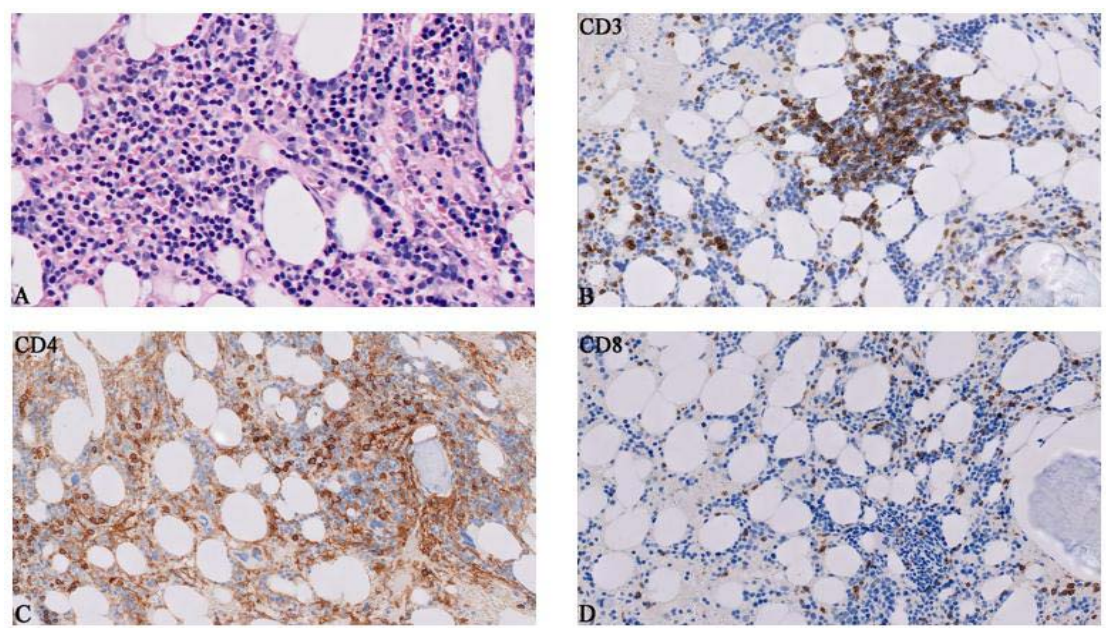

Figure 3: Histopathological findings in bone marrow at 6 months after initiation of pembrolizumab. A) Hematoxylin-eosin staining of trephine biopsy demonstrating a normocellular marrow with severe reduction of mature neutrophils (400x). B-D) Immunohistochemical stains for CD3, CD4, and CD8 showing T cell predominant lymphocytic infiltrate (200x).
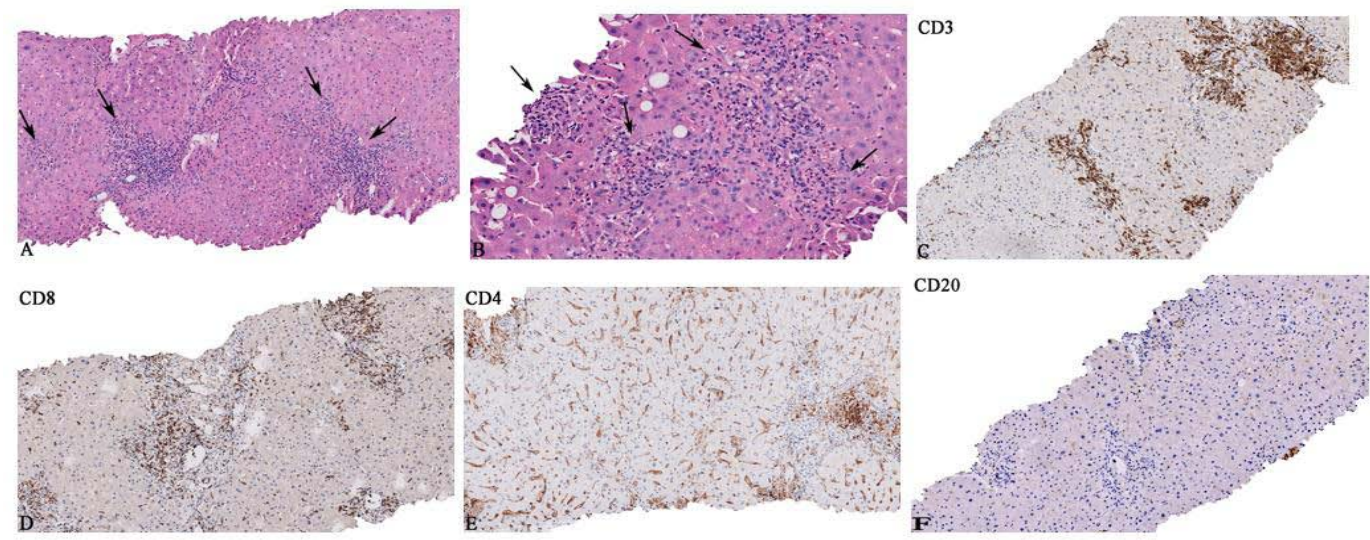

Figure 4: Histopathological changes in the liver biopsy at 9 months after initiation of pembrolizumab. A) Lobular hepatitis pattern with numerous foci of lymphohistiocytic lobular inflammation (arrows) (100x). B) Small and large non-caseating granulomas (arrows) (200x). C-F) Lobular and portal inflammatory infiltrates predominantly consisted of $\mathrm{CD}^{+}$and $\mathrm{CD} 8^{+}$lymphocytes, while fewer $\mathrm{CD} 4^{+}$and occasional CD20+ lymphocytes were seen (100x).

anti-CTLA-4 or other anti-PD-1 agent. Severe grade irAEs caused by anti-PD-1 monotherapy have a low incidence, ranging from $5 \%$ to $8 \%$ [1]. A meta-analysis of 19217 patients across 112 trials showed very low fatality rates of $0.36 \%$ among patients treated with anti-PD-1 monotherapy [2].

Immune-related hematological adverse events are amongst the rare complications of anti-PD-1 agents. A recent meta-analysis of 26 studies containing 5088 patients treated with anti-PD1 monotherapy reported an overall incidence of neutropenia and thrombocytopenia as low as $1 \%$ and $2 \%$ respectively [3]. The exact mechanism of Immune Checkpoint Inhibitors (ICPI) induced BM toxicity is unknown, a T cell mediated effect and an antibody driven process is proposed [4]. The striking feature in this case during neutropenia was a marked increase in all $\mathrm{T}$-cell subsets $\left(\mathrm{CD}^{+}, \mathrm{CD}^{+}\right.$, and $\mathrm{CD} 8^{+}$cells) on $\mathrm{FC}$ and a T-cell-predominant lymphocytic infiltrate in trephine biopsy. These findings in the absence of ANA suggest a T-cell mediated immune toxicity, which was supported by a good clinical response to G-CSF and corticosteroid.
Immune mediated thrombocytopenia is most likely to occur within the first 12 weeks of initiation of ICPI. Similar to our case, few reports describe a delayed onset of thrombocytopenia after discontinuation of the treatment $[5,6]$. Our literature search revealed that granulomatous reactions have been documented in the lung, liver, skin, mediastinal lymph nodes and colon induced by ICPI $[7,8]$. To the best of our knowledge this is the first documented case of granulomatous reaction after PD-1 inhibitor therapy, representing an ongoing immunologic reaction inducing thrombocytopenia in the BM.

Hepatic irAEs are commonly observed as asymptomatic elevations of AST and ALT levels [9]. Severe hepatitis of grade 3-4 is an uncommon but potentially serious toxicity of PD-1 inhibitors, which has been reported in $2 \%$ of CRC patients treated with pembrolizumab monotherapy [10]. Onset of hepatotoxicity typically occurs in 5 to 7 weeks of initiation of ICPI therapy $[9,11]$, however these can be seen as a late toxicity, even after discontinuation of therapy [12]. In this case we observed as late as 39 weeks post-infusion 


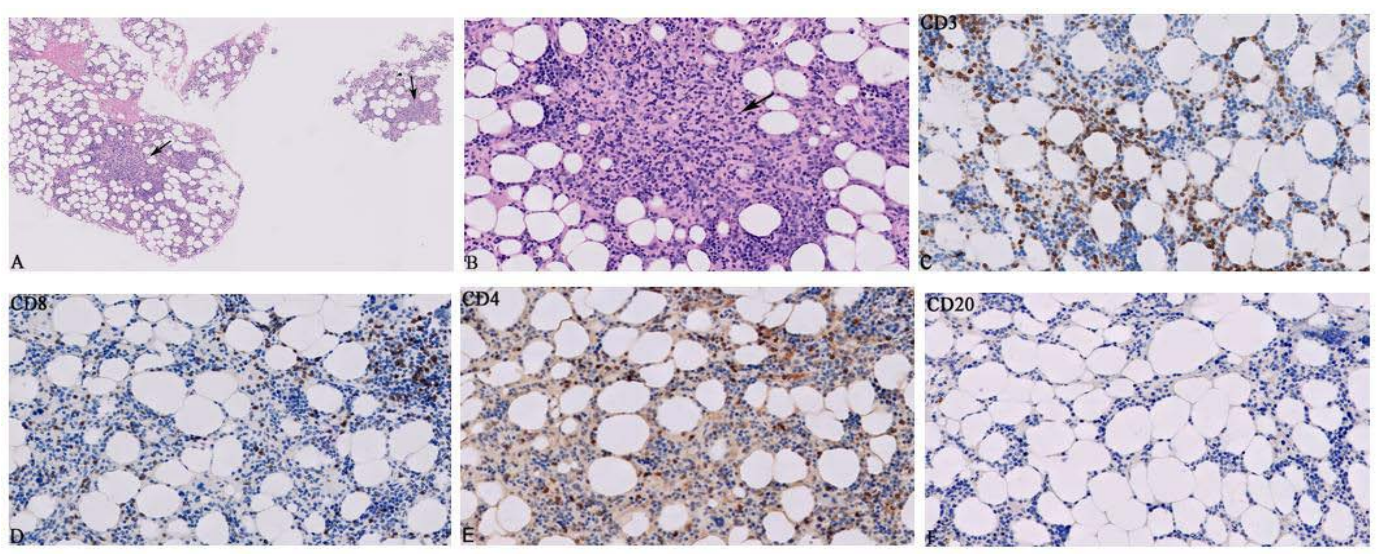

Figure 5: Histopathological changes observed in bone marrow at 9 months after initiation of pembrolizumab. A) Hematoxylin-eosin staining of trephine biopsy showing hypercellular marrow with granulomas (arrows) (50x). B) Non-caseating granulomas with reactive lymphocytosis at higher magnification (arrow) (200x). C-F) Immunoprofiling of the BM infiltrating T and B-Lymphocytes. CD3 and CD8 positive lymphocytes are significantly more than CD4 and CD20 positive lymphocytes (200x).

of pembrolizumab a mixed hepatocellular/cholestatic pattern of liver injury with significantly elevated transaminases above 8 folds and "direct" hyperbilirubinämie as high as $5.9 \mathrm{~g} / \mathrm{dl}$. Before attributing these symptoms to PD1 inhibitor therapy clinically, other causes were considered. The possibility of a voriconazole-related hepatotoxicity was considered in the differential diagnosis, but thought to be less likely as there was near normalization of his LFT after its dose reduction.

The reports on histopathological findings in ICI-related hepatitis are scare [13-15]. The most commonly described histological pattern includes lobular hepatitis, zone 3 hepatitis, marked portal lymphoid infiltrates and variable degrees of hepatic cell necrosis $[13,14]$. The liver biopsy of our patient revealed a pattern of acute lobular hepatitis with non-caseating granulomatous reaction. This pattern has also been described in a recent case series [15]. A recent study by Zen et al., focused on the immunophenotype of lymphocyte subsets and observed a predominance of $\mathrm{CD}^{+}$and $\mathrm{CD}^{+}$lymphocytes on immunostaining in ICPI. Liver biopsy findings of our patient also showed a predominance of $\mathrm{CD}^{+}$and $8+$ lymphocytes with a few $\mathrm{CD}^{+}$and $\mathrm{CD} 20^{+}$lymphocytes. Interestingly we also observed a similar infiltrate of increased $\mathrm{CD}^{+}$and $\mathrm{CD} 8^{+} \mathrm{T}$ cells along with granulomatous reaction in his BM biopsy in the clinical setting of thrombocytopenia. These findings suggest the possibility of an increased cytotoxic $\mathrm{T}$ cell response in organs affected by irAEs which in turn augment delayed-type hypersensitivity reaction with granulomatous inflammation.

Most reported cases of immune-mediated hepatitis resolve with termination of the ICPI or prompt application of immunosuppressive therapy [16,17]. In contrast, our patient eventually died from a rapid progression of acute hepatic failure. It might be possible that the concomitant voriconazole-related toxicity, may have contributed to the severe deterioration of his liver function.

\section{Conclusion}

In conclusion, our reported case contributes to the clinical and morphological spectrum of irAEs in patients treated with anti-PD-1 agents. The immunohistological characterization of the morphological findings plays an important role in confirming the diagnosis in severe toxicities associated with ICPIs and can facilitate in rapid initiation of the appropriate treatment. This case also highlights the delayed and severe grade immune mediated toxicities of pembrolizumab monotherapy with a fatal outcome. It illustrates the importance of clinical surveillance and management even after termination of antiPD1 therapy.

\section{References}

1. Kumar V, Chaudhary N, Garg M, Floudas CS, Soni P, Chandra AB. Current Diagnosis and Management of Immune Related Adverse Events (irAEs) Induced by Immune Checkpoint Inhibitor Therapy. Front Pharmacol. 2017; 8: 49.

2. Wang DY, Salem J, Cohen JV, et al. Fatal Toxic Effects Associated with Immune Checkpoint Inhibitors: A Systematic Review and Meta analysis. JAMA Oncol. 2018; 4: 1721-1728.

3. Sui JD, Wang $Y$, Wan $Y, W u$ YZ. Risk of hematologic toxicities with programmed cell death-1 inhibitors in cancer patients: a meta-analysis of current studies. Drug Des Devel Ther. 2018; 12: 1645-1657.

4. Finkel I, Sternschuss M, Wollner M, Shamai S, Peled N, Turgeman I, et al. Immune-related neutropenia following treatment with immune checkpoint inhibitors. J Immunother. 2020; 43: 67-74.

5. Delanoy N, Michot JM, Comont T, Kramkimel N, Lazarovici J, Dupont R, et al. Haematological immune-related adverse events induced by anti-PD-1 or antiPD-L1 immunotherapy: a descriptive observational study. Lancet Haematol. 2019; 6: e48-e57.

6. Atay $\mathrm{MH}$, Kelkitli E, Yilmaz B. Delayed severe thrombocytopenia due to Ipilimumab. J. BUON. 2015; 20: 1641-1642.

7. Danlos FX, Pages C, Baroudjian B, Vercellino L, Battistella M, Mimoun M, et al. Nivolumab-Induced Sarcoid-Like Granulomatous Reaction in a Patient With Advanced Melanoma. Chest. 2016; 149: e133-e136.

8. Rambhia PH, Reichert B, Scott JF, Feneran AN, Kazakov JA, Honda K, et al. Immune checkpoint inhibitor-induced sarcoidosis-like granulomas. Int. J. Clin. Oncol. 2019; 24: 1171-1181.

9. De Martin E, Michot J-M, Papouin B, Champiat S, Mateus C, Lambotte O, et al. Characterization of liver injury induced by cancer immunotherapy using immune checkpoint inhibitors. J Hepatol. 2018; 68: 1181-1190.

10. Le Dung T, Kavan $\mathrm{P}$, Kim TW, Burge ME, Cutsem EV, Hara $\mathrm{H}$, et al. KEYNOTE-164: pembrolizumab for patients with advanced microsatellite instability high (MSI-H) colorectal cancer. J Clin Oncol. 2018; 36: 3514-3514. 
11. Huffman BM, Kottschade LA, Kamath PS, Markovic SN. Hepatotoxicity afte immune checkpoint inhibitor therapy in melanoma: natural progression and management. Am J Clin Oncol. 2018; 41: 760-765

12. Couey MA, Bell RB, Patel AA, et al. Delayed immune-related events (DIRE) after discontinuation of immunotherapy: diagnostic hazard of autoimmunity at a distance. J Immunother Cancer. 2019; 7: 165.

13. Johncilla M, Misdraji J, Pratt DS, et al. Ipilimumab-associated Hepatitis: Clinicopathologic Characterization in a Series of 11 Cases. Am J Surg Pathol. 2015; 39: 1075-1084.

14. Zen Y, Yeh MM. Hepatotoxicity of immune checkpoint inhibitors: a histology study of seven cases in comparison with autoimmune hepatitis and idiosyncratic drug-induced liver injury. Mod Pathol. 2018; 31: 965-973.
15. Kleiner DE, Berman D. Pathologic changes in ipilimumab-related hepatitis in patients with metastatic melanoma. Dig Dis Sci. 2012; 57: 2233-2240.

16. Abdel-Rahman O, ElHalawani H, Fouad M. Risk of elevated transaminases in cancer patients treated with immune checkpoint inhibitors: a meta-analysis. Expert Opin Drug Saf. 2015; 14: 1507-1518.

17. Haanen JBAG, Carbonnel F, Robert C, Kerr KM, Peters S, Larkin J, et al. Management of toxicities from immunotherapy: ESMO Clinical Practice Guidelines for diagnosis, treatment and follow-up. Ann Oncol. 2017; 28: iv119-iv142. 